\title{
New Methods for Measuring the Thermal Emissivity of Semi-transparent and Opaque Materials
}

By D. Demange, M. Bejet, and B. Dufour

ONERA - DMSC - Fort de Palaiseau, Chemin de la Hunière 91761 Palaiseau, France CEDEX Tel: 33 (0) 1699361 23, Fax: 33 (0) 1699361 25, E-mail: demange@onera.fr

\begin{abstract}
Two original thermal emissivity measurement devices were developed recently in the laboratory. One of these devices uses a source as a means of heating a sample, and exhibits a certain number of advantages, including the absence of parasitic radiation on the surface studied, and the temperature homogeneity of the sample. This method was applied in measuring the emissivity of bulk ceramics and ceramic deposits on metallic or ceramic substrates. The second test facility, applied to opaque materials, measures the directional spectral emissivity and makes it possible to work back by calculation to the total hemispherical spectral emissivity. As an example, these two methods were used for characterizing thermal barriers for turbine vanes and materials that could be used in a High Temperature Reactor (HTR).
\end{abstract}

\section{Introduction}

In order to include internal or surface thermal radiation in codes predicting heating in insulating structures or in metallic systems where thermal radiation is preponderant, two original experimental devices was developed allowing the measurement of spectral thermal emissivity of semi-transparent materials and the directional spectral thermal emissivity of opaque materials. As an example, these two methods were used for characterizing thermal barriers for turbine vanes and materials that could be used in a High Temperature Reactor (HTR).

To ensure correct measurements on semi-transparent materials, one must avoid all spurious radiation such as black body radiation from a furnace for instance. The use of a laser source emitting at $10.6 \mu \mathrm{m}$ allows one to heat the sample without any stray radiation. The method used is to rotate a cylindrical sample about its axis and to expose a section of it to a high-power laser beam. The radiation emitted by the opposing section is then analysed by an FTIR spectrometer.

To measure the directional spectral emissivity of metals or more generally of opaque materials, a more standard apparatus has been set up. Heating of the specimen is carried out by a micro-furnace with electrical resistance. The samples are put into contact with one of the faces of a heating cylinder, and the whole apparatus

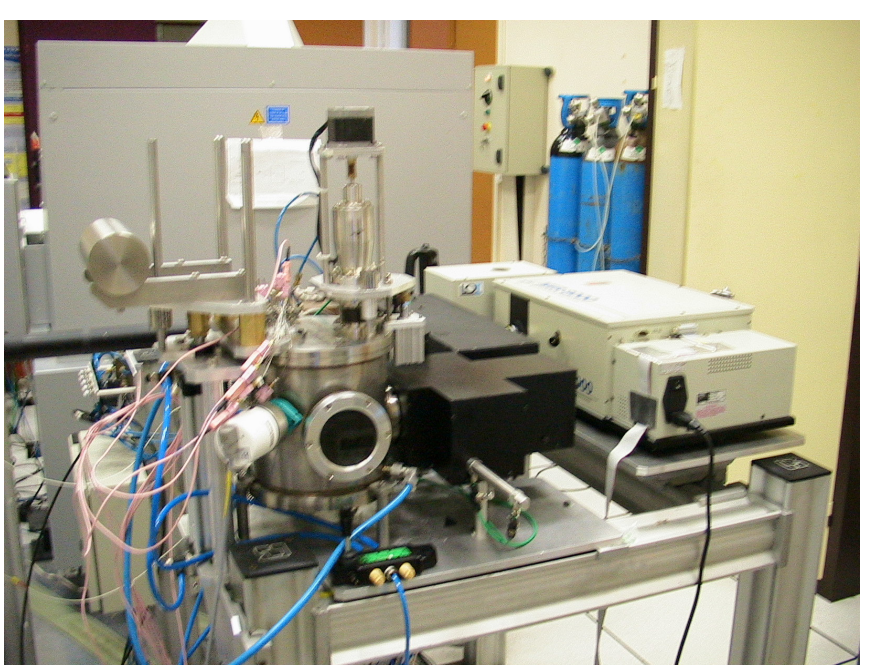

Figure 1-Overall view of the test facility. is put into a testing enclosure under a secondary vacuum $\left(10^{-5}\right.$ torr) to avoid all oxidation during the measurement. The nonpolluting micro-furnace is put into the enclosure and brings the sample to the measurement temperature.

\section{Description of experimental means}

The essential difference between the two testing means resides in the sample heating modes. For semi-transparent materials, the heating mode is a continuous power laser. This device is, however, limited to measuring the normal spectral emissivity of the materials and thus cannot be used to measure the directional emissivity. The total hemispherical emissivity thus cannot be measured. Another test facility was therefore developed to measure the directional spectral emissivity directly and to work back to the total hemispherical spectral emissivity by calculation. Here, the sample is heated by contact on one of the faces of a pivoting cylinder raised to the measurement temperature. Figure 1 shows the whole device. 


\subsection{Measuring spectral thermal emissivity in semi-transparent materials}

Knowing the thermo-radiative properties of semi-transparent materials is primordial for studies aimed at improving the insulating properties of thermal barriers or protections in the aerospace sector. The properties of these materials depend on the optic characteristics of the dense bulk material and on its porous or fiber texture. The important quantities are the normal or directional spectral thermal emissivity and the absorption and diffusion coefficients.

Kirchhoff's second law gives us the following relation:

$$
\varepsilon_{\lambda, T}=1-R_{\lambda, T}-T_{\lambda, T}
$$

In this expression $R_{\lambda, T}$ and $T_{\lambda, T}$ are the reflection and transmission coefficients normal to the sample. There exists a "Christiansen" wavelength where the coefficients $R_{\lambda_{c}, T}$ and $T_{\lambda_{c} T}$ are close to 0 and $\varepsilon_{\lambda_{c} T}$ is greater than 0.999 [4]. For wavelengths greater than the Christiansen wavelength, the transmission coefficient is close to 0 (figure 6) and we have $\varepsilon_{\lambda, T} \cong 1-R_{\lambda, T}$. The emissivity is independent of the material thickness. For wavelengths less than the Christiansen wavelength, the transmission coefficient will depend on the material's absorption and diffusion coefficient and on its texture. The emissivity may depend on the porosity and thickness of the sample being measured.

For emissivity measurements on semi-transparent materials, all parasitic radiation from the heating source (a furnace, for example) has to be avoided. A laser source can be used for heating if the emission wavelength is

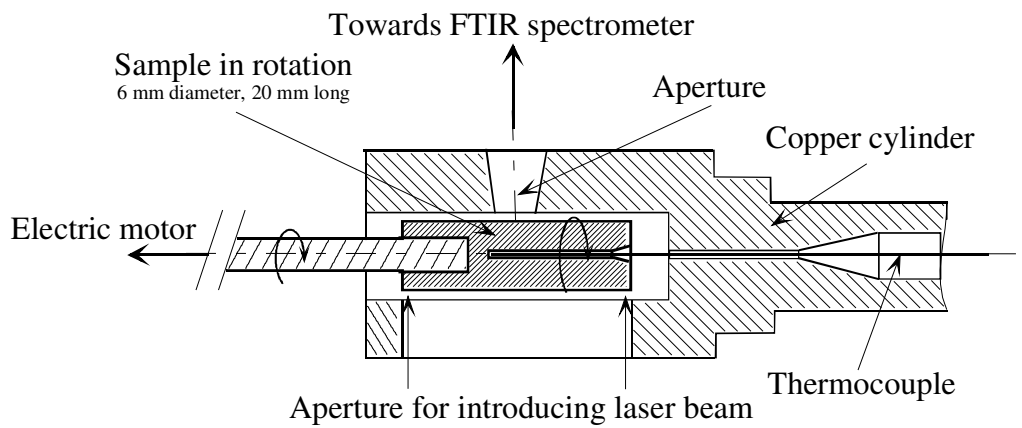

Figure 2 - View of the measurement cell within the material's highabsorptivity spectrum. $\mathrm{CO}_{2}$ lasers emit at $10.6 \mu \mathrm{m}$, which is within the wavelength domain $\left(\lambda_{c}\right)$ close to the Christiansen point. We developed a technique that consists of rotating a cylindrical sample (figure 2) about its axis and exposing a portion of it to a high-power laser beam. The radiation emitted by the portion on the opposing side is then analyzed by spectrometer with Fourier transform, operating in the mean infrared (2 to $25 \mu \mathrm{m}$ ).

The zone observed is

thus free of parasitic radiation (no furnace), and the sample rotation ensures lateral homogenization of the sample (external heating). Using a multi-mode laser ensures a rather good longitudinal homogeneity of the sample. With this technique, we can measure the absolute temperature of the sample with a thermocouple implanted at the center of the cylinder. A blackbody studied at Onera provides the spectral emission reference.

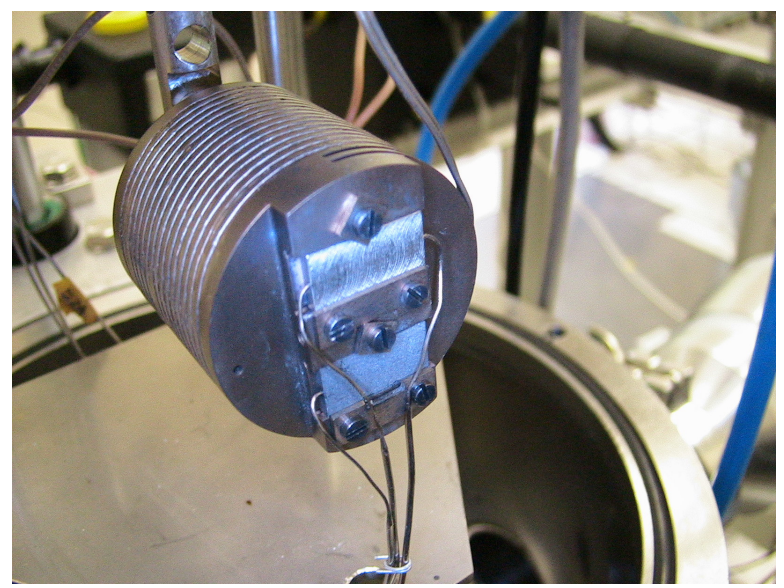

Figure 3 - Heating device - View of samples. 


\subsection{Measuring directional spectral thermal emissivity in opaque materials}

The principle consists in comparing the radiation emitted by the material's surface to that emitted by a

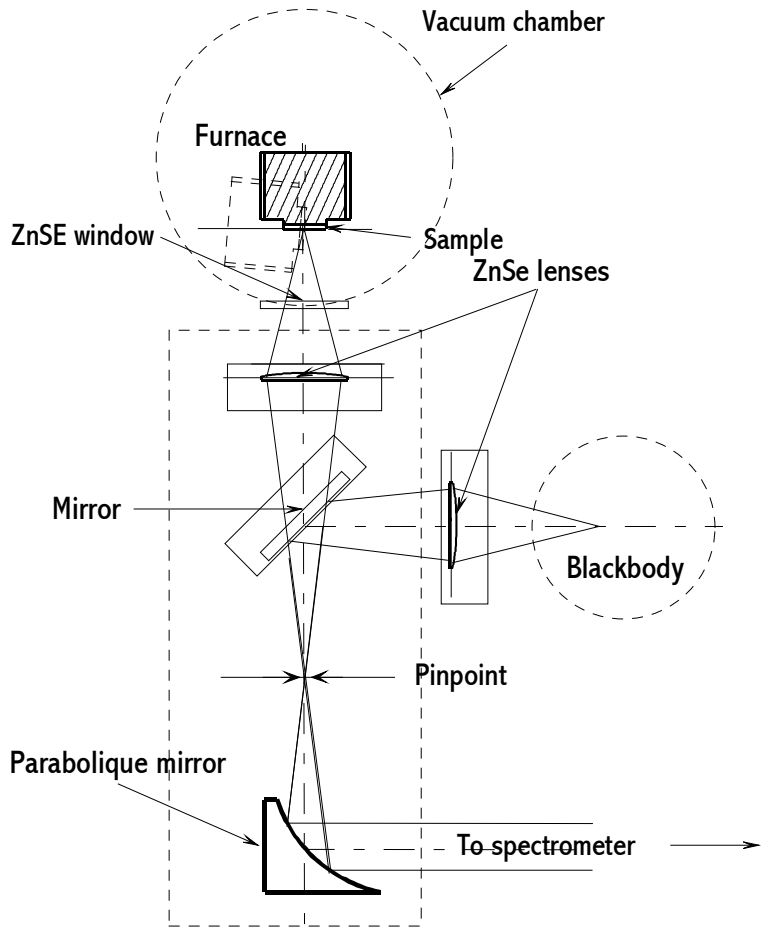

Figure4 - Overall view of the directional spectral thermal emissivity measurement rig. blackbody. Two samples are placed in a test chamber held at secondary vacuum $\left(<10^{-5}\right.$ torr) in order to avoid any oxidation during the measurement. A massive cylinder of non-polluting Inconel is raised to temperature by a Thermocoax electric resistor. The two samples are plated on one of the sides of the cylinder and are raised to the measurement temperature $\left(200^{\circ} \mathrm{C}\right.$ to $\left.850^{\circ} \mathrm{C}\right)$.

The sample is oriented at an incident angle $\theta$ that can be varied from $0^{\circ}$ to $85^{\circ}$ with respect to the normal. It is raised to temperatures between $250^{\circ} \mathrm{C}$ and $850^{\circ} \mathrm{C}$. The directional spectral luminance is measured with the same Fourier transform spectrometer as in the previous method. A Pyrox blackbody provides the spectral emission reference. The surface temperature is measured by two $\mathrm{N}$ type thermocouples implanted in the center of the sample, and a correction is made for the material's internal thermal gradient.

Figure 4 summarizes the principle of the whole measurement device.

A cooled cylindrical vacuum chamber is made in stainless steel. It is equipped with a pumping device to achieve a vacuum of better than $10^{-5}$ Torr. A step motor is mounted on the top of the chamber to rotate the furnace during the tests and thus allow measurements for incidence angles between 0 and $85^{\circ}$. The sample is a parallelepiped $8 \mathrm{~mm}$ wide, 16 $\mathrm{mm}$ long and $2 \mathrm{~mm}$ thick. Two holes of $0.6 \mathrm{~mm}$ are provided in the direction parallel to the sample plane and $0.5 \mathrm{~mm}$ from the observed face, for the installation of two $\mathrm{N}$ type thermocouples (figure 3 ).

\subsection{Determination of emissivity}

For a given system, the spectrometer response $(s)$ depends only on the temperature (following a non-linear law) and the emissivity of the surface, which gives us:

$$
\varepsilon_{\lambda}=\varepsilon_{c n}\left(\frac{s_{\text {matériau }}}{s_{c n}}\right)_{\lambda}
$$

For a reference blackbody, we have $\varepsilon_{\text {référence }} \approx 1$.

Actually, the blackbody emissivity is always less than unity, and there may exist a temperature difference, even very small, between the $\mathrm{CN}$ and the sample, inducing a non-negligible error in the emissivity measurement. So a sample of pure alumina provides a second reference around $\lambda_{c}$ (Christiansen wavelength) where we have $\varepsilon_{\lambda_{c}} \cong 1$.

If $R_{\lambda}=\left(\frac{s_{\text {matériau }}}{s_{c n}}\right)_{\lambda}$ is the ratio of the signals $(s)$ generated by the IR spectrometer, and if we consider a global correction coefficient $k_{c}$ close to unity and independent of the wavelength, we then have:

$$
\varepsilon_{\lambda}=k_{c} R_{\lambda}
$$




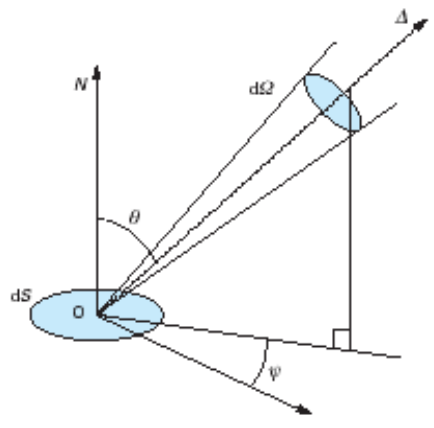

Figure 5 - Solid angle diagram
On sapphire, for $\lambda=\lambda_{\mathrm{c}}$ we get:

$$
k_{c}=\frac{\mathcal{E}_{\lambda_{c}}}{R_{\lambda_{c}}}
$$

If we allow $\varepsilon_{\lambda_{c}} \cong 1$ we will get $k_{c} \cong \frac{1}{R_{\lambda_{c}}}$.

The total directional emissivity is calculated by the following relation:

$$
\varepsilon_{\theta, T} \cong \frac{\int_{\lambda=\lambda_{\min }}^{\lambda_{\text {max }}} \varepsilon_{\lambda, T} L_{\lambda, T}^{0} d \lambda}{\int_{\lambda=\lambda_{\min }}^{\lambda_{\max }} L_{\lambda, T}^{0} d \lambda}
$$

The hemispherical spectral emissivity is then obtained by the relation $(d \omega=\sin \theta d \theta d \varphi)$ :

$$
\varepsilon_{\lambda, T}=\frac{1}{\pi} \int_{\Omega} \varepsilon_{\lambda, \theta} \cos \theta L_{\lambda, T}^{0} d \omega
$$

As well as the total hemispherical emissivity, which is equal to:

$$
\varepsilon_{T}=\frac{\int_{\lambda=\lambda_{\min }}^{\lambda=\lambda_{\max }} \varepsilon_{\lambda, T} L_{\lambda}^{0} d \lambda}{\int_{\lambda=\lambda_{\max }}^{\operatorname{man}} L_{\lambda, T}^{0} d \lambda}
$$

\section{DATA ANALYSIS}

\subsection{Measurement of alumina emissivity, comparison of data obtained by the two methods}

Figure 6 - Spectral emissivity of pure alumina (> 99.7\%) Comparison with two different methods

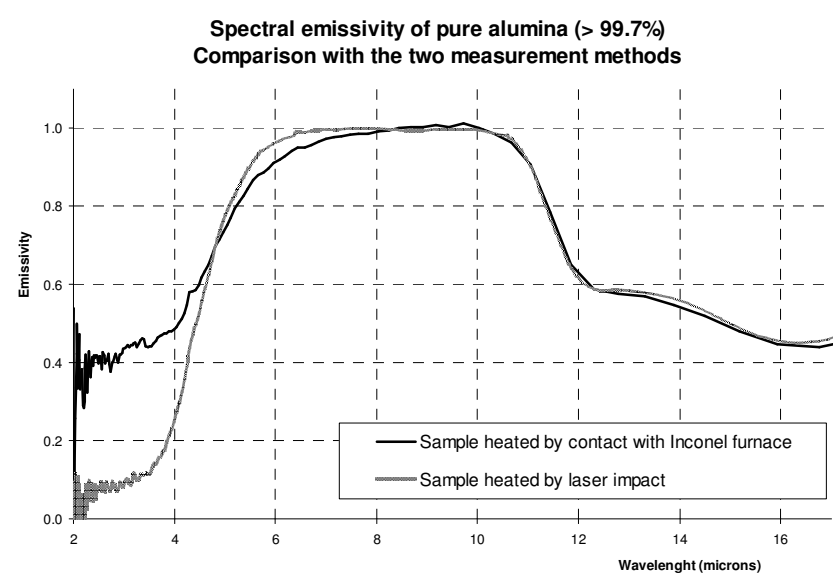

Data obtained on dense highpurity alumina $\left(>99,7 \% \mathrm{Al}_{2} \mathrm{O}_{3}\right)$ show the effect of the sample heating mode (method 1 or 2). Figure 6 shows three distinct regions. The first is a zone between wavelengths of 2 and $4.5 \mu \mathrm{m}$ where the emissivity is very different between the two methods and is much higher when the sample of $2 \mathrm{~mm}$ thickness is raised to temperature by contact with a heating cylinder. Here, the radiation emitted by the metallic surface is transmitted through the alumina and radiates outward, which gives us a high apparent emissivity. When heated by laser, only the material radiates and the emissivity is thus very weak despite a cylindrical sample $6 \mathrm{~mm}$ in diameter. In the second region between 4.5 and 11 $\mu \mathrm{m}$, the emissivity is much higher, the material's absorption coefficient increases, and for $\lambda=\lambda_{c}=9.5 \mu \mathrm{m}$ we have $\varepsilon_{\lambda_{c} T} \cong 1$ (Christiansen point). For wavelengths beyond $\lambda_{c}$, the material is opaque and we have $\varepsilon_{\lambda, T} \cong 1-R_{\lambda, T}$, which explains why we get the same emissivity for both methods. 


\subsection{Measurement of alumina emissivity, comparison of data obtained as a function of texture}

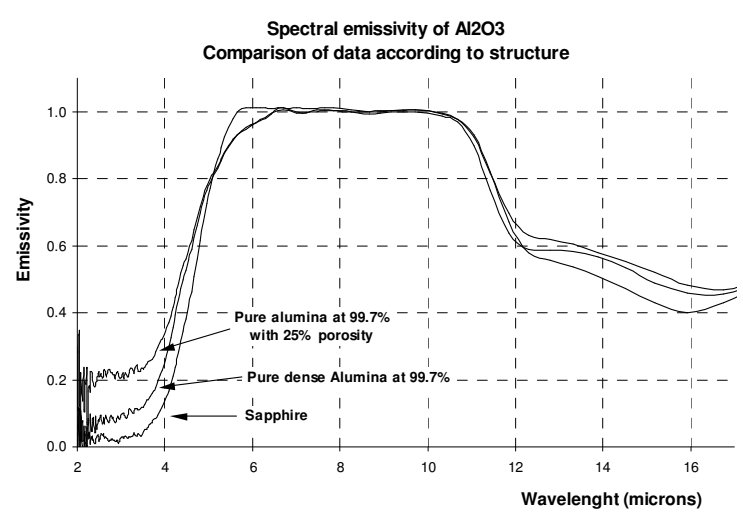

Figure 7 - Spectral emissivity of $\mathrm{Al}_{2} \mathrm{O}_{3}$ Comparison of data according to structure
The material's texture may have a nonnegligible effect on its emissivity. On aluminum oxide, $\mathrm{Al}_{2} \mathrm{O}_{3}$, figure 7 shows the low emissivity of sapphire (aluminum oxide single crystal) over a spectrum between 2 and $3 \mu \mathrm{m}$. High-purity polycrystalline alumina $\left(>99.7 \% \quad \mathrm{Al}_{2} \mathrm{O}_{3}\right)$ without porosity exhibits an emissivity of 0.08 over the same spectrum. A second alumina sample with $30 \%$ porosity shows its emissivity increased up to 0.2 .

\subsection{Measurement of directional spectral emissivity for metals}

Figure 8 gives an example of data obtained on stainless steel at $550^{\circ} \mathrm{C}$.

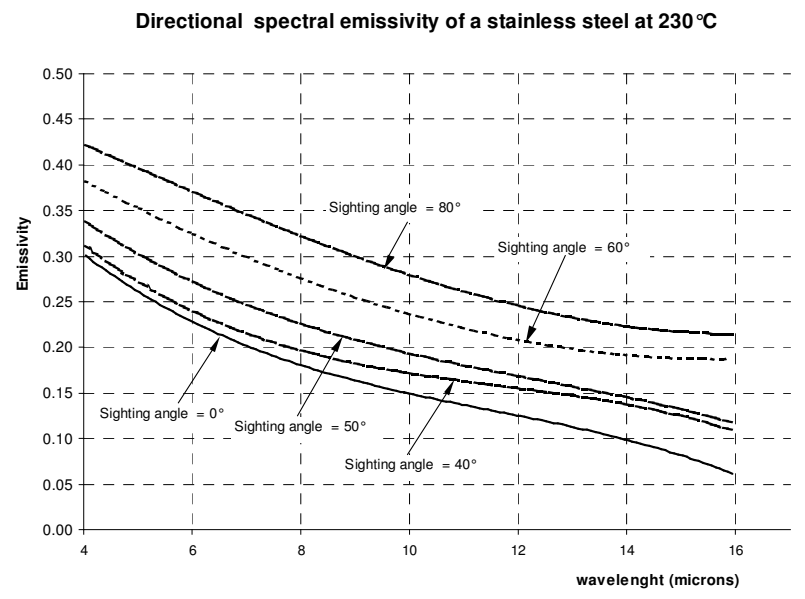

Figure 8 -Directional spectral emissivity of a stainless steel at $550^{\circ} \mathrm{C}$

We observe a decrease of the emissivity as a function of wavelength, and an increase as a function of observation angle.

\subsection{Example of application: Measurement of the specific heat of ceramics by flash method}

The principle of the method is to measure the temperature rise of a cylindrical sample after exposure of one of its faces to an energetic Dirac pulse. With the absolute rise in temperature and the known energy effectively absorbed by the sample, we can calculate the material's specific heat simply, by theory. An insulated sample of thickness $e$, mass $m$, specific heat $C p$, at temperature $\theta_{0}$, is exposed to a short pulse of energy density $(Q)$. After homogenization of its temperature it will undergo a maximum heating equal to:

$$
\theta_{\text {lim }}-\theta_{0}=T_{\lim }=\frac{Q}{\rho \cdot \mathrm{C}_{\mathrm{P}} \cdot \mathrm{e}}
$$

The specific heat can then be expressed by a relation of the form:

$$
C_{P} \propto \frac{T_{\max }^{*} U_{l a s} \cdot a_{\lambda_{l}} \varepsilon_{\lambda_{p}}}{m\left(U_{p y r}^{\max }-U_{p y r}^{0}\right)}
$$




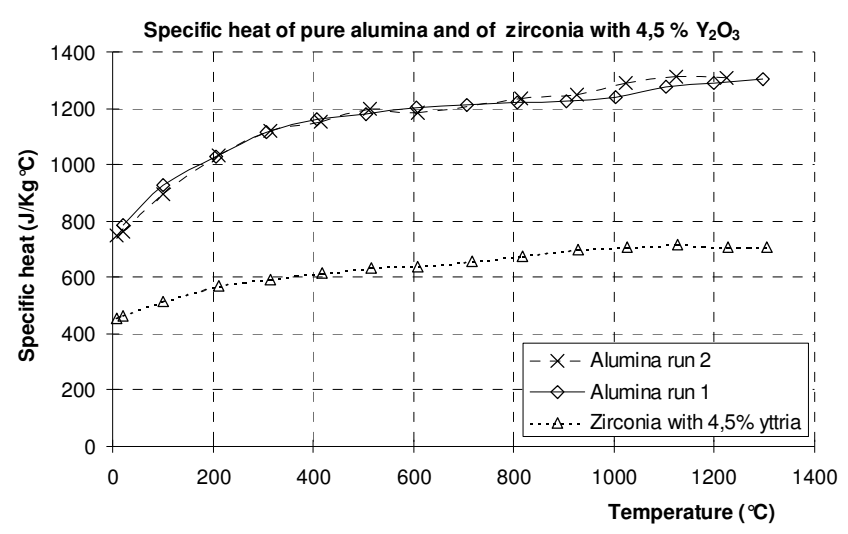

Figure 9-Measurement of the thermal diffusivity of an alumina and of the zirconium with $4.5 \% \mathrm{Y}_{2} \mathrm{O}_{3}$
In this expression, $U_{\text {las }}$ and $U_{p y r}$ represent the signals output by the laser calorimeter and by the pyrometer. The two coefficients

$a_{\lambda_{l}}$ et $\varepsilon_{\lambda_{p}}$ represent, respectively, the absorption coefficient under the laser beam and the emissivity over the pyrometer's sensitivity spectrum. $T_{\max }^{*}$ is the maximum reduced temperature [1], [2].

This method thus requires the knowledge of the product $a_{\lambda_{l}} \varepsilon_{\lambda_{p}}$. For ceramics, these two coefficients are close to unity for a wavelength range that is generally between 6 and $11 \mu \mathrm{m}$ (proximity of the Christiansen point). So this property allows us to do without these quantities as long as we use a laser source emitting on a domain of wavelength where the material has a high absorption, and a detector equipped with filters making it possible to obtain an adequate spectral response with the material's maximum emissivity range. The device for measuring the spectral thermal emissivity of semi-transparent materials, described in section 2.1, is very useful because it can be used to determine the value of the product $a_{\lambda_{l}} \varepsilon_{\lambda_{p}}$ and use relation (9) if this is very different from unity. Examples of data obtained on two ceramics are given in figure 9.

\section{Conclusion}

Two original thermal emissivity measurement devices were developed recently in the laboratory. One of these devices uses a source as a means of heating a sample, and exhibits a certain number of advantages, including the absence of parasitic radiation on the surface studied, and the temperature homogeneity of the sample. This method was applied in measuring the emissivity of bulk ceramics and ceramic deposits on metallic or ceramic substrates. The second test facility, applied to opaque materials, measures the directional spectral emissivity and makes it possible to work back by calculation to the total hemispherical spectral emissivity. The sample is heated by contact on one of the faces of a pivoting cylinder raised to the measurement temperature. This method is used for characterizing metallic or carbon-based materials and can measure the specific heat of ceramics by the flash method.

\section{Références}

[1] H. S. Carslaw, and J.C. Jaeger. Conduction of heat in solids. Oxford University Press (1959).

[2] D. Demange New application of the "flash" method. 15 th European on Thermophysical Properties (ECTP) High temperature - high pressures 2002, volume 34, pages 9-18

[3] R. Siegel and C. Spuckler, Analysis of thermal radiation effects on temperature in turbine engine thermal barrier coating - Materials Science Engineering A245 150-159 1998.

[4] B. Rousseau, J.F. Brun, D. de Sousa Meneses, and P. Echegut Temperature measurement : Christiansen and blackbody reference. International journal of thermophysics, vol. 26, $\mathrm{n}_{\mathrm{0}}$. 4, July 2005 